\title{
Brain-Dead and Coma Patients Exhibit Different Serum Metabolic Profiles: A Novel Diagnostic Approach in Neurocritical Care
}

\section{Tomasz Dawiskiba}

Wrocław Medical University

\section{Wojciech Wojtowicz}

Wrocław University of Science and Technology

\section{Badr Qasem}

Wrocław University of Science and Technology

Marceli Łukaszewski

Wrocław Medical University

Karolina Anna Mielko

Wrocław University of Science and Technology

Agnieszka Dawiskiba

4th Military Hospital of Wroclaw

Mirosław Banasik

Wrocław Medical University

Jan Paweł Skóra

Wrocław Medical University

Dariusz Janczak

Wrocław Medical University

Piotr Młynarz ( $\sim$ piotr.mlynarz@pwr.edu.pl)

Wrocław University of Science and Technology

\section{Research Article}

Keywords: brain death, coma patients, NMR spectroscopy, metabolomics analysis, multivariate data analysis, univariate data analysis

Posted Date: April 27th, 2021

DOl: https://doi.org/10.21203/rs.3.rs-451161/v1

License: (c) (1) This work is licensed under a Creative Commons Attribution 4.0 International License. Read Full License 
Version of Record: A version of this preprint was published at Scientific Reports on July 30th, 2021. See the published version at https://doi.org/10.1038/s41598-021-94625-3. 


\section{Abstract}

There is a clear difference between severe brain damage and brain death. However, in clinical practice, the differentiation of these states can be challenging. Currently, there are no laboratory tools that facilitate brain death diagnosis. The aim of our study was to evaluate the utility of serum metabolomic analysis in differentiating coma patients (CP) from individuals with brain death (BD). Serum samples were collected from 23 adult individuals with established diagnosis of brain death and 24 patients in coma with Glasgow Coma Scale 3 or 4, with no other clinical symptoms of brain death for at least 7 days after sample collection. Serum metabolomic profiles were investigated using proton nuclear magnetic resonance (NMR) spectroscopy. The results obtained were examined by univariate and multivariate data analysis (PCA, PLS-DA, and OPLS-DA). Metabolic profiling allowed us to quantify 43 resonance signals, of which 34 were identified. Multivariate statistical modeling revealed a highly significant separation between coma patients and brain-dead individuals, as well as strong predictive potential. The findings not only highlight the potential of the metabolomic approach for distinguishing patients in coma from those in the state of brain death but also may provide an understanding of the pathogenic mechanisms underlying these conditions.

\section{Introduction}

In classical terms, human death as a whole has been identified by the irreversible cessation of breathing and blood circulation [1], [2]. Shifting the boundary of death and extending it to patients with critical brain damage was revolutionary and changed the paradigms of modern medicine. It is assumed that it is impossible to determine either the beginning or the end of death, but medical criteria that most certainly confirm the irreversibility of this phenomenon should be provided at any time in its duration. The diagnosis of brain death in most cases can be based only on clinical examination with a detailed analysis of the causes, mechanisms and effects of brain damage [3]-[6]. Situations in which clinical tests allowing the diagnosis of brain death cannot be executed or clearly interpreted include cases of subtentorial brain damage, cases of extensive facial injuries, situations with the presence of abnormal neurological reflexes, and cases of being under the influence of certain contaminants or specific pharmacological agents [2], [7]. The diagnostic process must then be complemented by additional tests such as cerebral arteriography, which are not always easily accessible. It should be noted that laboratory diagnostics still have not proven to be a useful tool in such situations. Recognition of brain death, or at least suspicion of brain death, could resemble in such cases the diagnosis of acute myocardial infarction with the fundamental role of serum troponin determination. So far, the presumed potential of only two proteins has been initially disclosed S100B protein [8]-[10] and neuron-specific enolase NSE [9], [11], but these are single reports only, and no comprehensive analysis applying modern proteomic methods has yet been performed. It can therefore be concluded that, despite the seriousness of the problem, this theme is a kind of terra incognita in medicine, scientific reports dedicated to this subject are practically nonexistent, and the search for new methods of brain death diagnosis seems to be an absolute necessity. 
The concept of metabolomic studies is based on the observation that with the development of pathological processes, both local and systemic, the first symptoms appearing at the cellular level are directly reflected in the chemical composition of tissues subject to these processes and also in body fluids. These processes may be minor changes involving the disruption of the quantitative ratio between different chemical compounds as well as changes that are easier to detect, such as the appearance of entirely new chemical compounds (biomarkers of disease) or the disappearance of specific molecules present in homeostasis. The analysis of the abovementioned issue allows for the creation of predictive and discriminatory models by which the detection of even subtle changes in the concentrations of metabolites constitute the differentiating factor [12]-[14]. Metabolomics research is primarily based on gas or liquid chromatography - mass spectrometry (GC-MS/LC-MS) and nuclear magnetic resonance (NMR) platforms [12]-[15].

The aim of our research was to answer the question of whether there are changes in the profile of low molecular weight compounds present in blood serum in the process of brain death and to identify the metabolic biomarkers of this condition. This is the first such report in the literature.

\section{Materials And Methods}

\section{Serum sample collection}

Serum samples of brain-dead (BD) individuals and coma patients (CP) were collected mainly at the Department of Anesthesiology and Intensive Therapy of Wroclaw Medical University. Additionally, biological material from brain-dead cadavers was collected in other hospitals in the region of Lover Silesia during organ procurements performed by surgeons of the Department of Vascular, General and Transplantation Surgery of Wroclaw Medical University (the same protocol). The study was approved by the Commission of Bioethics at Wrocław Medical University (Approval no. KB-25/2018), and written informed consent was obtained from legal representatives of all the patients before enrollment in the study. All these research were conducted according to The Code of Ethics of the World Medical Association (Declaration of Helsinki).

The samples were collected from March 2018 to April 2019 from adult individuals with established diagnosis of brain death $(n=23)$ and comatose patients $(n=24)$ (Table 1). The diagnosis of brain death was determined and certified by three independent specialists according to current Polish law [16], [17] and was not related to the research project. The control group was formed of patients in coma in Glasgow Coma Scale 3 or 4, with no other clinical symptoms of brain death for at least 7 days after collection of serum (preservation of brain stem reflexes and/or of respiratory drive). No analgosedation was applied in the enrolled patients. The primary causes of brain death and coma were hypoxic-ischemic brain injury, traumatic brain injury and nontraumatic intracerebral hemorrhage (detailed information in Table 1). Patients with other causes of death or coma (e.g., brain tumors or meningitis) were excluded from the study, as well as those below 18 years, cases with evidence of malignancy or xenobiotic intoxication and individuals subjected to renal replacement therapy. No pregnant women were enrolled in 
the study. Signals related to medications (mannitol, furosemide, proton-pump inhibitors, antibiotics, noradrenaline, steroids, low molecular weight heparins and antipyretics) were eliminated from the statistical and chemometric evaluation.

Serum was sampled from the peripheral vein and collected using serum vacuum tubes (BD Vacutainer ref. 369032) that were then centrifuged at $1000 \times$ rpm for 15 minutes at $4{ }^{\circ} \mathrm{C}$. The samples were stored in Eppendorf-type tubes and kept at $-80^{\circ} \mathrm{C}$ until analysis. Transport was accomplished with the use of liquid nitrogen storage dewars.

Table 1. Demographic data and clinical profile of patients

\begin{tabular}{|lll|}
\hline & Brain-dead individuals & Coma patients \\
\hline Number of patients - overall & 23 & 24 \\
\hline Hypoxic-ischemic brain injury & 5 & 8 \\
\hline Traumatic brain injury & 4 & 8 \\
\hline $\begin{array}{l}\text { Nontraumatic intracerebral } \\
\text { hemorrhage }\end{array}$ & 14 & 8 \\
\hline GCS 3/GCS 4 & $23 /$ none & $13 / 11$ \\
\hline $7 / 30 / 90$ days survival & none & $24 / 18 / 13$ \\
- number of patients & & $14 / 10$ \\
\hline Sex (male/female) & $17 / 6$ & $56.7(19-87)$ \\
\hline Average age (mean/range) & $49.3(22-74)$ & \\
\hline
\end{tabular}

\section{Samples preparation and NMR measurements}

The collected serum samples were prepared according to a well-established protocol [18], [19]. The serum samples were thawed at room temperature and vortexed. Each serum sample $(200 \mu \mathrm{L})$ was mixed with $400 \mu \mathrm{L}$ of saline solution $(0.9 \% \mathrm{NaCl}, \mathrm{w} / \mathrm{v})$ containing $20 \% \mathrm{D}_{2} \mathrm{O}$ and centrifuged (10 min, $12000 \mathrm{RPM}, 4$ $\left.{ }^{\circ} \mathrm{C}\right)$. Supernatant $(550 \mu \mathrm{L})$ from each sample was transferred into a 5-mm NMR tube (SP, $5 \mathrm{~mm}$ ARMAR Chemicals). The samples were kept at $4^{\circ} \mathrm{C}$ before measurement.

The one-dimensional (1D) NMR spectra of serum samples were recorded at $298 \mathrm{~K}$ using an Avance II spectrometer (Bruker, GmBH, Germany) and cpmg1dpr pulse sequence with water presaturation (Bruker notation), which was operating at a proton frequency of $600.58 \mathrm{MHz}$. The serum sample spectra were collected as 128 following scans with spin-echo delay of $1000 \mu \mathrm{s}, 80$ loops, relaxation delay of $3.5 \mathrm{~s}$, 
acquisition time of $2.73 \mathrm{~s}$, size of FID (TD), 65536 points, spectra width of $20.01 \mathrm{ppm}$, line-broadening factor (LB), $0.3 \mathrm{~Hz}$ and transmitter frequency offset (01P), $4.722 \mathrm{ppm}$.

Two-dimensional (2D) NMR experiments were recorded and processed for selected samples. The performed experiments included ${ }^{1} \mathrm{H}-{ }^{1} \mathrm{H}$ correlation spectroscopy (COSY), total correlation spectroscopy (TOCSY), and ${ }^{1} \mathrm{H}^{13} \mathrm{C}$ heteronuclear single quantum correlation (HSQC).

\section{Processing of NMR spectra and resonance signal identification for data analysis}

The collected 1D ${ }^{1} \mathrm{H}$ NMR spectra were processed with LB of $0.3 \mathrm{~Hz}$ and manually phased and baseline corrected with MestReNova software (Mestrelab Research v 12.0.4). The spectral chemical shifts were referenced on the glucose anomeric carbon signal group $\delta=5.225 \mathrm{ppm}$. Spectral processing was performed in the $0.500 \mathrm{ppm}-10.000 \mathrm{ppm}$ chemical shift range. Spectral sections from 4.400 to 5.000 ppm, corresponding to water resonance signal suppression, were removed from the data matrix. The alignment of resonance signals was carried out with the use of the correlation optimized warping algorithm (COW) [20] and the icoshift algorithm implemented in MATLAB (v R2019a, Mathworks Inc.) [20]. All of the spectra were normalized by the PQN (probabilistic quotient normalization) method [21]. The calculation of the relative integral of NMR measured metabolites was obtained as a sum of data points of the nonoverlapping resonances or a cluster of partly overlapping resonances from the data matrix consisting of 46842 data points for each spectrum in $n$ dimensions. The third quartile values of the noise region $(0.625 \mathrm{ppm})$ were subtracted from the calculated relative integrals to decrease the influence on the final values.

The ${ }^{1} \mathrm{H}$ NMR resonance signals and corresponding chemical shifts were analyzed with statistical total correlation spectroscopy (STOSCY) [22] and identified in accordance with assignments published in the literature. Chenomx software (v 8.4 Chenomx Inc. Edmonton, Alberta, Canada) and online databases: Biological Magnetic Resonance Data Bank [23] (www.bmrb.wisc.edu) and Human Metabolome Data Base [24] (www.hmdb.ca).

\section{Univariate and multivariate data analysis}

All univariate data analyses were carried out with MATLAB software (v R2019a, Mathworks Inc.) on nonscaled data. Values below the limit of quantification were replaced with the third quartile value of the noise region $(0.625 \mathrm{ppm})$ for a specific variable. Levene's test was used to assess homogeneity of variation. Normality of distribution was verified with the Shapiro-Wilk test. Depending on the results of normality and variance tests, a parametric (equal/unequal variance Student's t-test) or nonparametric (Mann-Whitney-Wilcoxon test) variant was calculated. Multigroup univariate analysis was performed with the Kruskal-Wallis test with Dunn-Sidak post hoc tests. The false discovery rate (FDR) based on the Benjamini-Hochberg procedure was applied for the tested variables. All univariate statistical tests were calculated at a significance level of $a=0.05$. Dispersion of variables was represented by the coefficient of variation (CV) for normally distributed data or otherwise by the coefficient of quartile variation (QCV). 
Assessment of classifier performance was represented by ROC curve analysis using the perfcurve MATLAB (v R2019a, Mathworks Inc.) function.

The model calculations were performed using unit variance (UV) scaling for the relative integral values, while Pareto (Par) scaling was applied to a data matrix containing data from the entire spectrum. Both data sets were then used for calculations in the SIMCA 15.0.2 program (Sartorius Stedim Biotech, 2017). Principal component analysis (PCA) was applied for data overview and for extreme outlier detection based on Hotelling's T2 range (99\%). Discriminant analysis was performed by the partial least squares method (PLS-DA) for relative integral data (43 variables corresponding to the range of specific resonance signals) and orthogonal partial least squares (OPLS-DA) for whole spectra data (46842 variables as matching data points for chemical shifts). OPLS-DA data visualizations are presented together with Hotelling's T2 range (95\%) ellipse. The reliability of the PLS-DA and OPLS-DA models was assessed by cross validation analysis of variance (CV-ANOVA) at a significance level $a=0.05$. Discrimination model score plots are also presented as cross-validated versions in the supplementary materials [25]. The prediction effectiveness of the PLS-DA model is presented by a receiver operating characteristic (ROC) curve with the use of the perfcurve function in MATLAB (v R2019a, Mathworks Inc.). For this purpose, the true negative rate and true positive rate were determined according to sample class assignation, applying the 7-fold cross-validated predicted values from the modeled observations using the YPredcv function for the PLS-DA model (implemented in SIMCA 15.0.2 software, Sartorius Stedim Data Analytics AB).

\section{Results}

The ${ }^{1} \mathrm{H}$ NMR spectra measurements allowed us to quantify 43 resonance signals: 34 identified and 9 unknown compounds. Two data types were used to assess the possible distinction between the studied groups: the first based on the abovementioned specifically selected quantified resonance signals (Figs. 1 and 2) and the second with 46842 data points (Fig. 3). Details of the chemical shifts and resonance signals taken for analysis are presented in the supplementary data (Table S1).

The data obtained were elaborated by univariate and multivariate analysis. The exploratory analysis that was accomplished with the use of a PCA model based on the relative integrals of resonance signals revealed spontaneous separation between the studied groups (CP vs BD), although its extent was not substantial. In addition, PCA based on the Hotelling T2 range with a significance level equal to 0.01 revealed no extreme outliers, enabling the use of all observations for discriminant analysis based on PLS and OPLS models. The variables that influenced the positions of observations in the PCA model are presented in Figure 1.

Both calculated multivariate models demonstrated favorable separation between the CP and BD groups, attained high predictive potential and were strongly statistically relevant (Table 2). The PLS-DA model validation based on cross-validated analysis of variance (CV-ANOVA) revealed statistical significance with a $p$ value $=1.56 \mathrm{E}-07$, while the OPLS-DA model had a $\mathrm{p}$ value $=4.54 \mathrm{E}-05$. The PLS-DA discriminant model was also assessed with the use of a receiver operating characteristic curve (ROC) and the area 
under the curve value (AUC). Its sensitivity and specificity were calculated from sample class prediction during the cross-validation procedure (YpredCV) in SIMCA v15.0.2 software. The discriminant model obtained a high AUC value equal to 0.944. The graphical representations for PLS-DA and OPLS-DA comparisons between the $\mathrm{CP}$ and $\mathrm{BD}$ groups for the two latent variable models are shown in Figures 2 and 3. To verify the influence of resonance signals that were not quantified, the OPLS-DA model based on whole-spectra analysis was calculated along with a graphical representation of the importance of the variables in the predictive component versus the chemical shift (Fig. 3, right). The graph is colored according to model loadings scaled as coefficients of correlation between the models and original data (Fig. 3, right).

Verification of potentially important single variables as valuable classifiers between coma patients and brain-dead individuals proceeded with the use of univariate analysis. Statistically important metabolites are presented together with descriptive statistics and ROC curve (AUC) values (Table 3). Univariate analysis revealed significant differences in 15 resonance signals, of which 9 were identified according to chemical shift, STOCSY analysis and 2D NMR spectra, while 6 remained unidentified. For those statistically important metabolites, fold change values were calculated (Fig. 4), as well as their AUC values (Fig. 5). Seven metabolites were increased in the BD group (Unk 1, Unk 5, methanol, acetone, Unk 2, acetate, 3-methyl-2-oxovalerate), and 8 were decreased (Unk 7, isoleucine, betaine, Unk 6, methylhistidine, glycine, Unk 9 valine). The highest change was observed in the relative integral of the singlet resonance signal for methanol, with a fold change equal to 11.13. Of the abovementioned statistically important metabolites, 5 exhibited AUC values above 0.800 (Unk 1, Unk 5, methanol, Unk 7 and acetone), including 2 values even exceeding 0.900 (Unk 5 and methanol). The whole-spectra analysis by the OPLS-DA model confirmed the results of PLS-DA, indicating the two most important ${ }^{1} \mathrm{H}$ NMR spectra with $\mathrm{p}$ (corr) $>0.700$ at $1.15 \mathrm{ppm}$ and $3.34 \mathrm{ppm}$ (corresponding to Unk 1 and methanol, respectively). The response permutation testing plots for the PLS-DA and OPLS-DA models are presented in the supplementary data (Fig. S1).

Table 2. Main model parameters from PLS-DA for comparison between coma patients and brain-dead individuals.

\begin{tabular}{|llllllll|}
\hline Comparison & $\begin{array}{l}\text { Model } \\
\text { type }\end{array}$ & $\mathbf{N}$ & PC/LV & R2X(cum) & R2Y(cum) & Q2(cum) & $\begin{array}{l}\text { p value } \\
\text { (CV- } \\
\text { ANOVA) }\end{array}$ \\
\hline $\begin{array}{l}\text { CP vs BD (Relative } \\
\text { integral) }\end{array}$ & PCA & 47 & 2 & 0.279 & - & - & - \\
\hline $\begin{array}{l}\text { CP vs BD (Relative } \\
\text { integral) }\end{array}$ & PLS-DA & 47 & 2 & 0.254 & 0.792 & 0.562 & $\begin{array}{l}1.56 \mathrm{E}- \\
07\end{array}$ \\
\hline CP vs BD (Spectra) & OPLS-DA & 47 & $1+4+0$ & 0.671 & 0.914 & 0.613 & $\begin{array}{l}4.54 \mathrm{E}- \\
05\end{array}$ \\
\hline
\end{tabular}


Table 3. Univariate analysis for quantified ${ }^{1} \mathrm{H}$ NMR resonance signals compared between coma patients and brain-dead individuals. The metabolite order is based on ascending adjusted $p$ values. Calculations made on ${ }^{(1)}$ mean or ${ }^{(2)}$ median; ${ }^{*} \mathrm{CV}$ (coefficient of variation), ${ }^{*} \mathrm{QCV}$ (quantile coefficient of variation); ${ }^{a} \mathrm{t}$ test for equal variances, ${ }^{b}$ t-test for unequal variances, ${ }^{c}$ Mann-Whitney-Wilcoxon test

\begin{tabular}{|c|c|c|c|c|c|}
\hline \multirow[t]{3}{*}{ Metabolite } & \multirow[t]{3}{*}{$\begin{array}{l}\text { Fold change } \\
\text { (BD/CP) }\end{array}$} & $\begin{array}{l}\text { CV or } \\
\text { QCV }\end{array}$ & $\begin{array}{l}\text { CV or QCV } \\
{[\%)}\end{array}$ & $\begin{array}{l}\text { FDR } \\
\text { adjusted }\end{array}$ & \multirow[t]{3}{*}{ AUC } \\
\hline & & {$[\%)$} & $\mathrm{CP}$ & p-value & \\
\hline & & BD & & & \\
\hline $\begin{array}{l}\text { Unk } 1 \text { (1.158 ppm) } \\
\text { (d) }\end{array}$ & $1.62^{(1)}$ & 25.57 \# & $30.51^{\#}$ & $2.01 \mathrm{E}-05^{\mathrm{a}}$ & 0.893 \\
\hline $\begin{array}{l}\text { Unk } 5(2.060 \mathrm{ppm}) \\
(\mathrm{s})\end{array}$ & $2.25^{(1)}$ & $39.90 \#$ & $16.79^{\#}$ & $2.01 \mathrm{E}-05^{\mathrm{b}}$ & 0.906 \\
\hline Methanol & $11.13^{(1)}$ & $73.25 \#$ & 44.00 \# & $7.67 \mathrm{E}-05^{\mathrm{b}}$ & 0.913 \\
\hline $\begin{array}{l}\text { Unk } 7 \text { (2.903 ppm) } \\
(\mathrm{m})\end{array}$ & $0.56^{(1)}$ & $28.00 \#$ & $38.03{ }^{\#}$ & $1.39 \mathrm{E}-04^{\mathrm{b}}$ & 0.870 \\
\hline Acetone & $2.13^{(2)}$ & $35.25^{*}$ & $18.05^{*}$ & $9.65 \mathrm{E}-04^{\mathrm{C}}$ & 0.830 \\
\hline Unk 2 (1.169) (t) & $1.95^{(2)}$ & $16.67^{*}$ & $23.75^{*}$ & $5.75 \mathrm{E}-03^{c}$ & 0.786 \\
\hline Acetate & $1.97^{(2)}$ & $61.22^{*}$ & $39.90^{*}$ & $1.30 \mathrm{E}-02^{\mathrm{C}}$ & 0.759 \\
\hline Isoleucine & $0.88^{(2)}$ & $23.58^{*}$ & $18.36^{*}$ & $1.30 \mathrm{E}-02^{\mathrm{C}}$ & 0.759 \\
\hline Betaine & $0.69^{(1)}$ & $26.90^{\#}$ & $43.51^{\#}$ & $1.51 \mathrm{E}-02^{b}$ & 0.725 \\
\hline $\begin{array}{l}\text { 3-Methyl-2- } \\
\text { oxovalerate }\end{array}$ & $1.43^{(1)}$ & $37.20 \#$ & $42.48^{\#}$ & $1.51 \mathrm{E}-02^{\mathrm{a}}$ & 0.754 \\
\hline $\begin{array}{l}\text { Unk } 6 \text { (2.148 ppm) } \\
\text { (s) }\end{array}$ & $0.36^{(2)}$ & $44.73^{*}$ & $45.53^{*}$ & $1.51 \mathrm{E}-02^{\mathrm{C}}$ & 0.745 \\
\hline Methylhistidine & $0.75^{(2)}$ & $18.15^{*}$ & $12.29^{*}$ & $1.51 \mathrm{E}-02^{\mathrm{C}}$ & 0.745 \\
\hline Glycine & $0.41^{(2)}$ & $45.27^{*}$ & $47.42^{*}$ & $2.32 \mathrm{E}-02^{\mathrm{C}}$ & 0.728 \\
\hline $\begin{array}{l}\text { Unk } 9 \text { (7.138 ppm) } \\
\text { (d) }\end{array}$ & $0.41^{(2)}$ & $60.74^{*}$ & $52.23^{*}$ & $2.32 \mathrm{E}-02^{\mathrm{C}}$ & 0.728 \\
\hline Valine & $0.80^{(1)}$ & $24.78^{\#}$ & $32.13^{\#}$ & $3.50 \mathrm{E}-02^{\mathrm{a}}$ & 0.745 \\
\hline
\end{tabular}


Our ${ }^{1} \mathrm{H}$ NMR-based metabolomic approach demonstrated serum metabolic differences between patients in coma and individuals with diagnosis of brain death. The results obtained showed favorable separation and model parameters in cross-validated PLS-DA and OPLS-DA models with high predictability in both relative integral and whole spectra data for the studied sample. The discrimination potential between groups was also confirmed for specific metabolites with high ROC AUCs. The findings not only establish the potential of metabolomics in neurocritical care but may also provide an understanding of the pathogenic mechanisms underlying brain death. This is the first scientific publication in the literature relating to metabolomic studies of brain death.

In recent years, a few research projects aimed at metabolomic assessment of clinical conditions associated with central nervous system pathologies have been conducted; the goal in most cases was to identify diagnostic biomarkers for stroke or traumatic brain injury. The use of multivariate statistical analysis has made it possible to demonstrate significant separation between patients with cerebral pathology and healthy individuals in most of the studies [15], [26], [35]-[37], [27]-[34]. For example, in the case of stroke the group of potential biomarkers includes, among others: lactate [27], [29], [31], [32], pyruvate [27], [29], [32], glycolate [27], formate [27], glutamine [27], methanol [27], acetate [29], [32], cysteine [26], folic acid [26], S-adenosyl homocysteine [26], oxidized glutathione [26], tyrosine[15], [30], [31], tryptophan [31], serine[30]-[32], isoleucine [28], [30], valine [15], [28], [30], [32], glycine [32], leucine [15], [28], betaine [30], [32], carnitine [15], [30] and ketone bodies (acetone, acetoacetate and $\beta$ hydroxybutyrate) [29] as blood biomarkers; citrate [27], hippurate [27], and glycine [27] as urine biomarkers; and finally acetic acid [38], 3-hydroxyisovaleric acid [38], 3-hydroxybutyric acid [38], choline [38], glycine [38], pyruvic acid [38], I-lactic acid [38], acetone [38] and branched chain amino acids (valine, leucine, isoleucine) [28] as cerebrospinal fluid biomarkers. Notably, in selected research projects, the adopted targeted metabolomic analysis proved to be helpful in differentiating cerebral infarction (Cl) patients and those with intracerebral hemorrhage (ICH) [15], [30]. Of course, the discriminatory power of the above-listed metabolites varied; however, statistical significance was maintained in each case. Similarly, favorable results have been obtained when using metabolomics in the analysis of states of traumatic brain injury (TBI), although far fewer research projects have been conducted here. The performed studies have shown that proline [33], phosphoric acid [33], $\beta$-hydroxybutyric acid [33], galactose [33], creatinine [33], valine [33], linoleic acid [33], arachidonic acid [33], medium-chain fatty acids (decanoic and octanoic acids) [34] and sugar derivatives including 2,3-bisphosphoglyceric acid [34] in the blood; as well as propylene glycol [35], lactate [35], [36], glutamine [35], creatine [35] and glutamate [36] in the cerebrospinal fluid should be considered potential markers of acute TBI and could even serve as death predictors [36]. As stated before, there are no reports in the literature on metabolomics in braindead patients; however, a few research projects using magnetic resonance spectroscopy and magnetic resonance imaging have been performed to study in vivo metabolic changes in brain tissue. The conclusions from these studies have been that high levels of lactate, choline and lipids and decreased levels of n-acetyl aspartate are prognostic factors of brain death [39]-[41]. It has also been demonstrated that magnetic resonance spectra in these individuals are dominated by intense inorganic phosphate 
signals and are characterized by a complete absence of adenosine triphosphate (ATP) and phosphocreatine at the same time [42], [43].

Our results demonstrate that there are metabolites that can be considered potential biomarkers of brain death. The metabolomic serum analysis comparing brain-dead individuals to patients in coma revealed statistically significant increases in the concentrations of methanol, acetone, acetate and 3-methyl-2oxovalerate and simultaneous statistically significant decreases in the concentrations of isoleucine, betaine, methylhistidine, glycine and valine. There were also significant changes in the concentrations of other metabolites that played significant roles in discrimination, although we were unable to identify them unambiguously (Table 3). All resonance signals underwent the primary identification procedure using statistical total correlation spectroscopy (STOCSY) and a two-dimensional NMR spectroscopic approach (analysis accuracy level 2 based on Sumer et al. [44]. The unidentified resonance signals were determined by their multiplicity patterns and chemical shifts. To precisely identify them, advanced methods of serum purification should be carried out, and then mass spectrometry (MS) analysis should be performed. We plan to accomplish these steps in the next stage of research.

It is not possible to compare the results of our research with the analyses of other authors, as no studies on metabolomics in brain death have been conducted so far. Moreover, we did not make comparisons with healthy people but with comatose patients, which prevents the results of our research from being compared to analyses known from the literature of other brain pathologies, where healthy individuals were always the reference points. As a result, a lower concentration of an individual metabolite in our comparisons does not exclude its being higher than in healthy persons and vice versa. In the case of brain death, it should also be noted that this condition affects not only brain tissue but also the functioning of the entire organism in a more extensive way than does any other brain pathology.

Among the identified metabolites that allowed us to discriminate individuals in the state of brain death from comatose patients, methanol featured the greatest statistical significance. Methanol is a naturally occurring compound in normal, healthy individuals and is formed through anaerobic fermentation by intestinal bacteria [45]-[47] and metabolic processes involving S-adenosylmethionine, highly localized in the pituitary gland [45], [48]. It is widely recognized as a putative causative agent of neurodegenerative diseases [45], [49]-[51]; however, it has been found to be decreased in stroke patients [27]. The increase in methanol levels in the brain death state may be related to intestinal paralysis commonly occurring in this condition, followed by increased bacterial translocation, as well as due to insufficiency of metabolic clearance mechanisms that physiologically maintain low levels of methanol in the organism [45]. Ketone bodies such as acetone, acetoacetate and beta-hydroxybutyrate are the source of energy for the brain when glucose is less available [29], [38] and could provide up to $60 \%$ of the human brain's metabolic needs [52]. The effect of this is the increase in acetone concentration observed not only in the plasma of patients with ischemic stroke [29] but also in their cerebrospinal fluid [38]. The administration of ketones has even been shown to provide neuroprotection after brain hypoxia/ischemia or traumatic brain injury [52], [53]. Elevated acetone in individuals with brain death compared to patients in coma is a result of the obvious extreme energy deficiencies of the brain tissue in such cases. The same also applies to acetate, 
which in our research turned out to be another metabolite at increased concentrations. Under normal resting conditions, acetate may contribute up to $10-15 \%$ of the basal energy demands of brain astrocytes [54]-[56], so its upregulation may be important for meeting bioenergetic demands in a critical state. On the other hand, a significant reduction in acetate uptake and utilization by astrocytes in cerebral ischemia [57], [58], as well as its formation by $\mathrm{N}$-acetylaspartate hydrolysis during brain infarction [59], have been described, possibly contributing to acetate elevation in the state of brain death. Our observations indicating the discriminatory potential of acetone as a biomarker of brain death are indirectly confirmed by the results of autopsy studies on animals, which have shown its significant postmortem increase in brain tissue [59], [60]; however, it is also elevated in the ischemic core and penumbra after cerebral infarction [58]. The last metabolite identified to be significantly elevated in braindead individuals compared to comatose patients in our study was 3-methyl-2-oxovalerate. This abnormal metabolite arising mainly from the incomplete breakdown of isoleucine is a well-known neurotoxin that plays a critical role in neurological damage [61], [62].

Isoleucine and valine, the concentrations of which decrease significantly in the state of brain death compared to coma, are branched chain amino acids (BCAAs) that participate in the synthesis of brain cell signaling molecules such as serotonin, dopamine and norepinephrine and influence brain function by the production of energy via the citric acid cycle (CAC) [30], [63]. Reduction in levels of isoleucine [28], [30], [31] and valine [28], [31], [32] is commonly observed in ischemic stroke; moreover, it is described as associated with infarction severity and poor neurological outcome [28]. It has also been observed in several critical illnesses such as traumatic brain injury, sepsis, burn injury and heart diseases [28], [30], [31], [64], [65]. It is presumed that BCAA depletion could arise from their utilization as amine neurotransmitters or CAC intermediates for brain function recovery processes [30], and it is also a possible cause of their even more profound reduction in the state of brain death. A similar situation is related to the observation of changes in glycine. This simplest amino acid is one of the major inhibitory neurotransmitters in the central nervous system [66], and its release might be inhibited as a consequence of neural excitation during brain ischemia and injury [31]. High levels of glycine protect neuronal cells during brain ischemia [66], [67], whereas a decrease in glycine has a detrimental effect on ischemic neuronal damage [66], [68]. The downregulation of glycine in serum is commonly presented in the literature of stroke [28], [30], [31]; however, there is also one report describing the opposite observation [32]. Betaine is another metabolite whose significant decrease in concentration differentiates the state of brain death from coma in our observations and, as in the previous case, the literature describes its changes in other brain pathologies in different ways (an increase [30] as well as a decrease [32] in serum of patients in stroke). Betaine is hypothesized to influence pathways of inhibitory neurotransmitter production and recycling [69], but most of all, it is a protective substance that helps to reduce [32], [70] high levels of homocysteine - a well-documented promoter of ischemic injury to endothelial cells in brain vessels, an oxidative stress inductor, and a compound that influences the pro-thrombotic system [71]. Betaine-dependent protective mechanisms seem to be devastated in cerebral destruction processes [32]. The explanation of the drop in the concentration of the last compound with discriminatory potential that we identified, methylhistidine, is not as obvious as in the cases of the previously described metabolites. 
Approximately $75 \%-90 \%$ of this histidine derivative is formed in skeletal muscles [65], [72]-[74]; therefore, its measurement (especially in urine and less frequently in plasma) is a commonly used biomarker for skeletal muscle protein breakdown [65], [73], [75], [76]. Considering the above, it is not surprising that the urine excretion of methylhistidine in sepsis [77], thermal injuries [78] and traumatic brain injury [79] has been found to be increased, indicating the upregulation of skeletal muscle catabolism in critical states of the organism. On the other hand, it has also been shown that an elevation of the urinary concentration of methylhistidine can occur, with an accompanying paradoxical decrease in plasma, as demonstrated in patients with Alzheimer's disease [80]. We believe that the reduction in methylhistidine concentration in our study could indicate the relative downregulation of skeletal muscle catabolism in individuals with brain death in comparison to coma patients, remembering that in both of these states, increased catabolic processes are of course unquestionably present.

Medicine does not have any laboratory tests that are able to confirm brain death, so this condition is diagnosed only on the basis of clinical examination, optionally complemented by instrumental methods, which are not always easily accessible. From this point of view, the results of our study demonstrating the potential of ${ }^{1} \mathrm{H}$ NMR-based metabolic serum fingerprinting with multivariate metabolomic data analysis are particularly valuable. Further studies in this field should not only be regarded as constituting a great scientific challenge but also as a necessity for modern medicine, especially intensive care and transplantation medicine.

\section{References}

1. De Georgia, M. A. History of brain death as death: 1968 to the present. J. Crit. Care. 29, 673-678 (2014).

2. Gardiner, D., Shemie, S., Manara, A. \& Opdam, H. International perspective on the diagnosis of death. British Journal of Anaesthesia. 108, i14-i28 (2012).

3. Scripko, P. D. \& Greer, D. M. An Update on Brain Death Criteria. Neurologist. 17, 237-240 (2011).

4. Spinello, I. M. Brain Death Determination. Journal of Intensive Care Medicine. 30, 326-337 (2015).

5. Wijdicks, E. F. M. Determining brain death in adults. Neurology. 45, 1003-1011 (1995).

6. Wijdicks, E. F. M. The case against confirmatory tests for determining brain death in adults. Neurology. 75, 77-83 (2010).

7. Drake, M., Bernard, A. \& Hessel, E. Brain Death. Surgical Clinics of North America. 97, 1255-1273 (2017).

8. Li, D. R. et al. Postmortem serum protein S100B levels with regard to the cause of death involving brain damage in medicolegal autopsy cases. Leg. Med. 8, 71-77 (2006).

9. Rodríguez-Rodríguez, A. et al. S100B and Neuron-Specific Enolase as mortality predictors in patients with severe traumatic brain injury. Neurol. Res. 38, 130-137 (2016).

10. Shakeri, M., Mahdkhah, A. \& Panahi, F. Posttraumatic Brain Death Predictor Biomarkers; S100B Protein Levels In Accosiation With Patients Outcome. Arch. Trauma Res. 2, 76-80 (2013). 
11. Suzuki, Y. et al. Prolonged elevation of serum neuron-specific enolase in children after clinical diagnosis of brain death. Journal of Child Neurology. 27, 7-10 (2012).

12. Nicholson, J. K., Lindon, J. C. \& Holmes, E. 'Metabonomics': Understanding the metabolic responses of living systems to pathophysiological stimuli via multivariate statistical analysis of biological NMR spectroscopic data. Xenobiotica. 29, 1181-1189 (1999).

13. Nicholson, J. K. et al. Metabolic phenotyping in clinical and surgical environments. Nature. 491, 384392 (2012).

14. Fiehn, O. Metabolomics - The link between genotypes and phenotypes. Plant Mol. Biol. 48, 155-171 (2002).

15. Hu, Z. et al. Rapid and Sensitive Differentiating Ischemic and Hemorrhagic Strokes by Dried Blood Spot Based Direct Injection Mass Spectrometry Metabolomics Analysis. J. Clin. Lab. Anal. 30, 823830 (2016).

16. (No Title). Available at: https://www.monitorpolski.gov.pl/M2007046054701.pdf. (Accessed: 12th April 2021)

17. Bohatyrewicz, R. et al. Reversal to Whole-Brain Death Criteria After 15-Year Experience With Brain Stem Death Criteria in Poland. Transplant. Proc. 41, 2959-2960(2009).

18. Dawiskiba, T. et al. Serum and urine metabolomic fngerprinting in diagnostics of inflammatory bowel diseases. World J. Gastroenterol. 20, 163-174 (2014).

19. Wojtowicz, W. et al. Serum and urine $1 \mathrm{H}$ NMR-based metabolomics in the diagnosis of selected thyroid diseases. Sci. Rep. 7, 1-13 (2017).

20. Savorani, F., Tomasi, G. \& Engelsen, S. B. icoshift: A versatile tool for the rapid alignment of 1D NMR spectra. J. Magn. Reson. 202, 190-202 (2010).

21. Dieterle, F., Ross, A., Schlotterbeck, G. \& Senn, H. Probabilistic quotient normalization as robust method to account for dilution of complex biological mixtures. Application in1H NMR metabonomics. Anal. Chem. 78, 4281-4290 (2006).

22. Cloarec, O. et al. Statistical total correlation spectroscopy: An exploratory approach for latent biomarker identification from metabolic 1 H NMR data sets. Anal. Chem. 77, 1282-1289 (2005).

23. Ulrich, E. L. et al. BioMagResBank. Nucleic Acids Res. 36, D402-D408 (2008).

24. Wishart, D. S. et al. HMDB 4.0: the human metabolome database for 2018. Nucleic Acids Res. 46, D608-D617 (2018).

25. Bevilacqua, M. \& Bro, R. Can We Trust Score Plots? Metabolites. 10, 278 (2020).

26. Jiang, Z. et al. A metabonomic approach applied to predict patients with cerebral infarction. Talanta. 84, 298-304 (2011).

27. Jung, J. Y. et al. 1 H-NMR-based metabolomics study of cerebral infarction. Stroke. 42, 1282-1288 (2011).

28. Kimberly, W. T., Wang, Y., Pham, L., Furie, K. L. \& Gerszten, R. E. Metabolite profiling identifies a branched chain amino acid signature in acute cardioembolic stroke. Stroke. 44, 1389-1395 (2013). 
29. Baranovicova, E. et al. NMR metabolomic study of blood plasma in ischemic and ischemically preconditioned rats: an increased level of ketone bodies and decreased content of glycolytic products $24 \mathrm{~h}$ after global cerebral ischemia. J. Physiol. Biochem. 74, 417-429 (2018).

30. Liu, P. et al. Discovery of Metabolite Biomarkers for Acute Ischemic Stroke Progression. J. Proteome Res. 16, 773-779 (2017).

31. Wang, D., Kong, J., Wu, J., Wang, X. \& Lai, M. GC-MS-based metabolomics identifies an amino acid signature of acute ischemic stroke. Neurosci. Lett. 642, 7-13 (2017).

32. Wang, Y., Wang, Y. G., Ma, T. F., Li, M. \& Gu, S. L. Dynamic metabolites profile of cerebral ischemia/reperfusion revealed by $1 \mathrm{H}$ NMR-based metabolomics contributes to potential biomarkers. Int. J. Clin. Exp. Pathol. 7, 4067-4075 (2014).

33. Zheng, F. et al. Plasma metabolomics profiles in rats with acute traumatic brain injury. PLoS One. 12, e0182025 (2017).

34. Orešič, M. et al. Human Serum Metabolites Associate With Severity and Patient Outcomes in Traumatic Brain Injury. EBioMedicine 12, 118-126(2016).

35. Glenn, T. C. et al. Metabolomic analysis of cerebral spinal fluid from patients with severe brain injury. Acta Neurochir. Suppl. 118, 115-119 (2013).

36. Stefani, M. A. et al. Elevated glutamate and lactate predict brain death after severe head trauma. Ann. Clin. Transl. Neurol. 4, 392-402 (2017).

37. Zhang, X. et al. Distinguishing intracerebral hemorrhage from Acute cerebral infarction through metabolomics. Rev. Investig. Clin. 69, 319-328 (2017).

38. Wang, Y. et al. 1H NMR-based metabolomics exploring biomarkers in rat cerebrospinal fluid after cerebral ischemia/reperfusion. Mol. Biosyst. 9, 431-439 (2013).

39. Garde, K. et al. Phosphorous and proton spectroscopy in relation to near incarceration and incarceration of the human brain. Acta radiol. 35, 197-200 (1994).

40. Falini, A. et al. Progressive brain failure after diffuse hypoxic ischemic brain injury: a serial MR and proton MR spectroscopic study.Am. J. Neuroradiol.19, (1998).

41. Monsein, L. H. The imaging of brain death. Anaesthesia and Intensive Care. 23, 44-50 (1995).

42. Kato, T. et al. Assessment of brain death in children by means of P-31 MR spectroscopy: Preliminary note: Work in progress. Radiology. 179, 95-99 (1991).

43. Aichner, F. et al. Magnetic resonance: A noninvasive approach to metabolism, circulation, and morphology in human brain death. Ann. Neurol. 32, 507-511 (1992).

44. Sumner, L. W. et al. Proposed minimum reporting standards for chemical analysis: Chemical Analysis Working Group (CAWG) Metabolomics Standards Initiative (MSI). Metabolomics. 3, 211221 (2007).

45. Dorokhov, Y. L., Shindyapina, A. V., Sheshukova, E. V. \& Komarova, T. V. Metabolic methanol: Molecular pathways and physiological roles. Physiol. Rev. 95, 603-644 (2015). 
46. Jensen, N. S. \& Canale-Parola, E. Bacteroides pectinophilus sp. nov. and Bacteroides galacturonicus sp. nov.: two pectinolytic bacteria from the human intestinal tract.Appl. Environ. Microbiol.52, (1986).

47. Siragusa, R. J., Cerda, J. J., Baig, M. M., Burgin, C. W. \& Robbins, F. L. Methanol production from the degradation of pectin by human colonic bacteria. Am. J. Clin. Nutr. 47, 848-851 (1988).

48. Axelrod, J. \& Daly, J. Pituitary gland: Enzymic formation of methanol from S-adenosylmethionine. Science (80-.). 150, 892-893 (1965).

49. Tong, Z. et al. Aging-associated excess formaldehyde leads to spatial memory deficits. Sci. Rep. 3, 1-9 (2013).

50. Tong, Z. et al. Urine formaldehyde level is inversely correlated to mini mental state examination scores in senile dementia. Neurobiol. Aging. 32, 31-41 (2011).

51. Yang, M. et al. Alzheimer's disease and methanol toxicity (Part 1): Chronic methanol feeding led to memory impairments and tau hyperphosphorylation in mice. J. Alzheimer's Dis. 41, 1117-1129 (2014).

52. Prins, M. L. Cerebral metabolic adaptation and ketone metabolism after brain injury. Journal of Cerebral Blood Flow and Metabolism. 28, 1-16 (2008).

53. Puchowicz, M. A. et al. Neuroprotection in diet-induced ketotic rat brain after focal ischemia. J. Cereb. Blood Flow Metab. 28, 1907-1916 (2008).

54. Killen, M. J., Giorgi-Coll, S., Helmy, A., Hutchinson, P. J. A. \& Carpenter, K. L. H. Metabolism and inflammation: implications for traumatic brain injury therapeutics. Expert Review of Neurotherapeutics. 19, 227-242 (2019).

55. Mashimo, T. et al. Acetate is a bioenergetic substrate for human glioblastoma and brain metastases. Cell. 159, 1603-1614 (2014).

56. Dienel, G. A. \& Cruz, N. F. Astrocyte activation in working brain: Energy supplied by minor substrates. Neurochem. Int. 48, 586-595 (2006).

57. Hosoi, R. et al. Sensitive Reduction in 14C-acetate Uptake in a Short-term Ischemic Rat Brain. J. Stroke Cerebrovasc. Dis. 16, 77-81 (2007).

58. Alf, M. F. et al. High-resolution spatial mapping of changes in the neurochemical profile after focal ischemia in mice. NMR Biomed. 25, 247-254 (2012).

59. Nonaka, M. et al. Decrease in N-acetylaspartate without commensurate accumulation of acetate in focal cerebral ischemia in rat. Neurol. Res. 21, 771-774 (1999).

60. Musshoff, F. et al. Comparison of post-mortem metabolic changes in sheep brain tissue in isolated heads and whole animals using 1 H-MR spectroscopy-preliminary results. Int. J. Legal Med. 125, 741-744 (2011).

61. Wang, T. et al. L-carnitine prevents ammonia-induced cytotoxicity and disturbances in intracellular amino acid levels in human astrocytes. J. Gastroenterol. Hepatol. 34, 1249-1255 (2019).

62. $\mathrm{Xu}, \mathrm{J}$. et al. Integrated UPLC-Q/TOF-MS Technique and MALDI-MS to Study of the Efficacy of YiXinshu Capsules Against Heart Failure in a Rat Model. Front. Pharmacol. 10, 1474 (2019). 
63. Fernstrom, J. D. Branched-chain amino acids and brain function. in Journal of Nutrition 135, 1539S1546S(American Institute of Nutrition, 2005).

64. Huang, Y., Zhou, M., Sun, H. \& Wang, Y. Branched-chain amino acid metabolism in heart disease: an epiphenomenon or a real culprit? Cardiovasc. Res. 90, 220-223 (2011).

65. Holeček, M. Relation between glutamine, branched-chain amino acids, and protein metabolism. in Nutrition 18, 130-133 (Elsevier Inc., 2002).

66. Yao, W. et al. Glycine exerts dual roles in ischemic injury through distinct mechanisms. Stroke. 43, 2212-2220 (2012).

67. Gusev, E. I. et al. Neuroprotective Effects of Glycine for Therapy of Acute Ischaemic Stroke. Cerebrovasc. Dis. 10, 49-60 (2000).

68. Ke, C., Pan, C. W., Zhang, Y., Zhu, X. \& Zhang, Y. Metabolomics facilitates the discovery of metabolic biomarkers and pathways for ischemic stroke: a systematic review. Metabolomics. 15, 152 (2019).

69. Knight, L. S., Piibe, Q., Lambie, I., Perkins, C. \& Yancey, P. H. Betaine in the Brain: Characterization of Betaine Uptake, its Influence on Other Osmolytes and its Potential Role in Neuroprotection from Osmotic Stress. Neurochem. Res. 42, 3490-3503 (2017).

70. Atkinson, W., Elmslie, J., Lever, M., Chambers, S. T. \& George, P. M. Dietary and supplementary betaine: acute effects on plasma betaine and homocysteine concentrations under standard and postmethionine load conditions in healthy male subjects. Am. J. Clin. Nutr. 87, 577-585 (2008).

71. Sidorov, E., Sanghera, D. K. \& Vanamala, J. K. P. Biomarker for ischemic stroke using metabolome: A clinician perspective. J. Stroke. 21, 31-41 (2019).

72. Nagasawa, T., Yoshizawa, F. \& Nishizawa, N. Plasma N т -Methylhistidine Concentration Is a Sensitive Index of Myofibrillar Protein Degradation during Starvation in Rats. Biosci. Biotechnol. Biochem. 60, 501-502 (1996).

73. Berridge, B. R., Van Vleet, J. F., Herman, E. \& Cardiac in Haschek and Rousseaux's Handbook of Toxicologic Pathology1567-1665(Elsevier Inc., 2013). Vascular, and Skeletal Muscle Systems doi:10.1016/B978-0-12-415759-0.00046-7

74. Holeček, M. Histidine in Health and Disease: Metabolism, Physiological Importance, and Use as a Supplement. Nutrients. 12, 848 (2020).

75. Sjölin, J. et al. Splanchnic and peripheral release of 3-methylhistidine in relation to its urinary excretion in human infection. Metabolism. 38, 23-29 (1989).

76. Lukaski, H. C., Mendez, J., Buskirk, E. R. \& Cohn, S. H. Relationship between endogenous 3methylhistidine excretion and body composition. Am. J. Physiol. - Endocrinol. Metab.3, (1981).

77. Long, C. L. et al. Urinary excretion of 3-methylhistidine: An assessment of muscle protein catabolism in adult normal subjects and during malnutrition, sepsis, and skeletal trauma. Metabolism. 30, 765776 (1981).

78. Bilmazes, C. et al. Quantitative contribution by skeletal muscle to elevated rates of whole-body protein breakdown in burned children as measured by Nt-methylhistidine output. Metabolism. 27, 
671-676 (1978).

79. Charrueau, C. et al. Metabolic response and nutritional support in traumatic brain injury: Evidence for resistance to renutrition. J. Neurotrauma. 26, 1911-1920 (2009).

80. Fonteh, A. N., Harrington, R. J., Tsai, A., Liao, P. \& Harrington, M. G. Free amino acid and dipeptide changes in the body fluids from Alzheimer's disease subjects. Amino Acids. 32, 213-224 (2007).

\section{Figures}
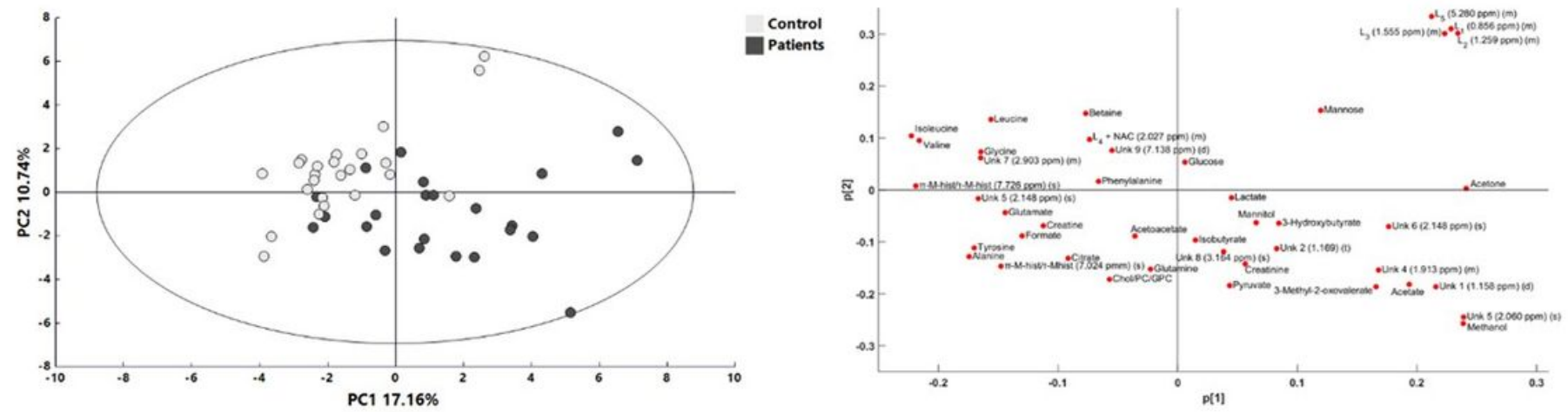

\section{Figure 1}

PCA model (left) and corresponding loading plot (right) with all measured variables for patients enrolled in the study. Light gray - coma patients (CP); gray - brain-dead individuals (BD).
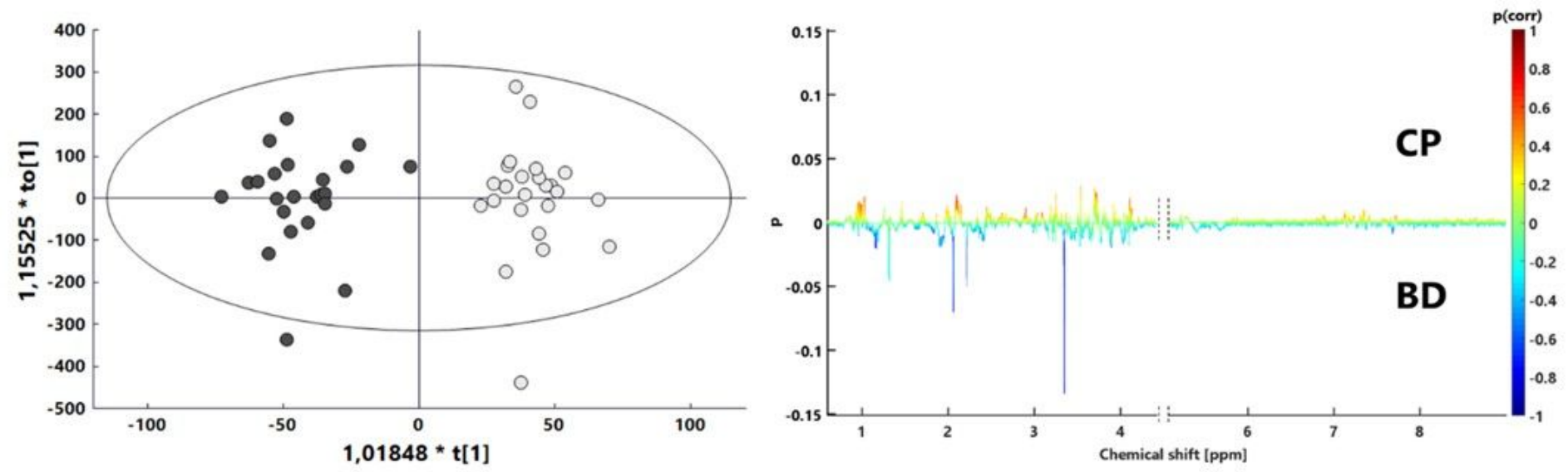

Figure 3

OPLS-DA score plot with corresponding s-line plot. Light gray - coma patients (CP); gray - brain-dead individuals (BD); red circle on the ROC curve marking the optimal operating point. 


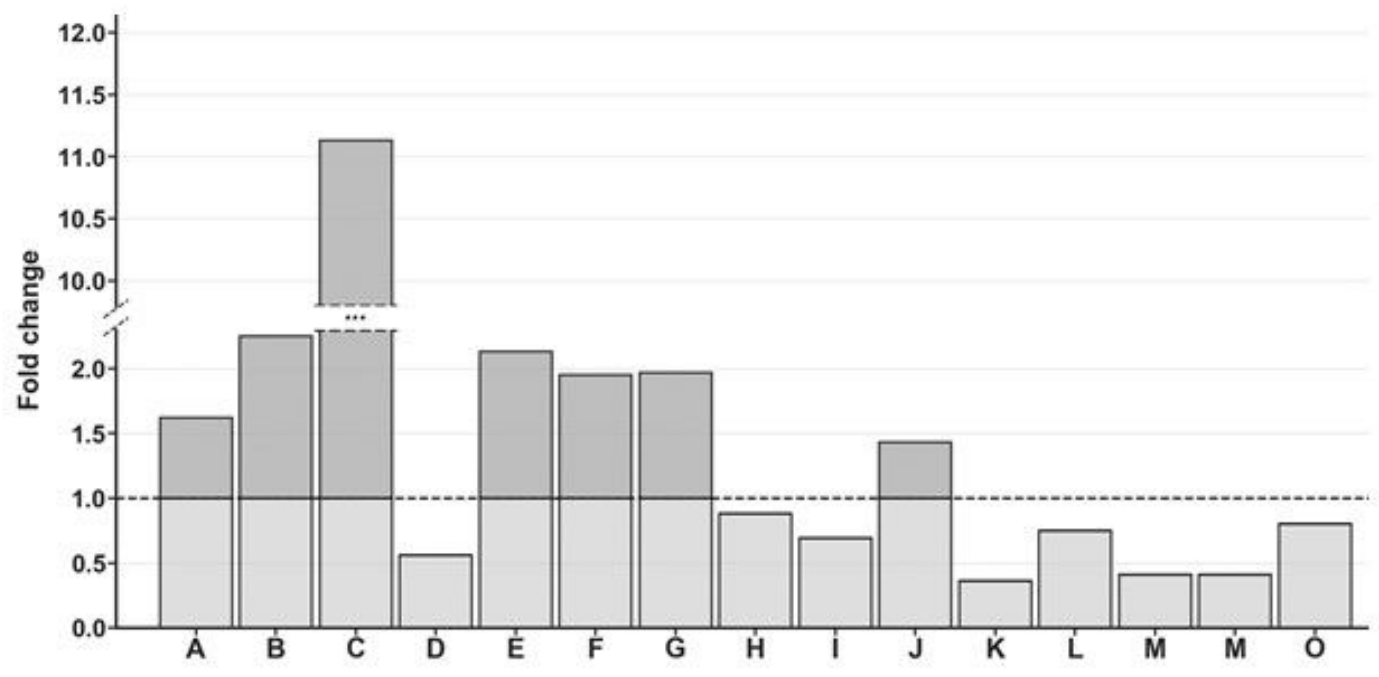

\section{Figure 4}

Fold change calculation among statistically important metabolites - brain-dead individuals vs coma patients. A - Unk 1 (1.158 ppm) (d), B - Unk 5 (2.060 ppm) (s), C - methanol, D - Unk 7 (2.903 ppm) (m), E acetone, $\mathrm{F}$ - Unk 2 (1.169) (t), G - acetate, $\mathrm{H}$ - isoleucine, I - betaine, J - 3-methyl-2-oxovalerate, $\mathrm{K}$ - Unk 6 (2.148 ppm) (s), L - methylhistidine (s), M - glycine, N - Unk 9 (7.138 ppm) (d), 0 - valine.
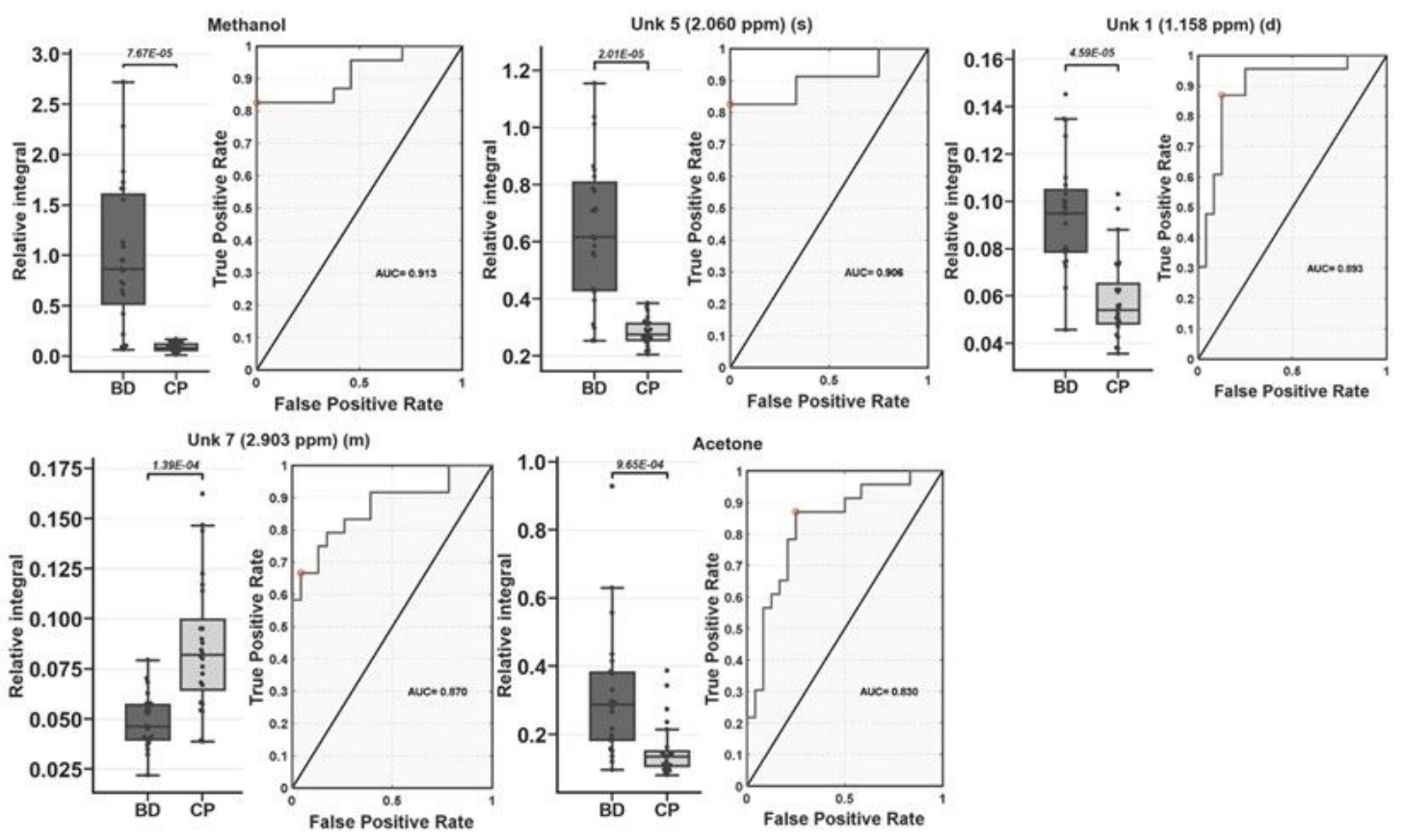

Figure 5 
Boxplots and ROC curves for statistically important metabolites with AUC $>0.800$. For boxplots: whiskers - 1.5x interquartile range (IQR); bar - average; box - range between first quartile (Q1) and third quartile (Q3). Red circle on ROC curve marking optimal operating point.

\section{Supplementary Files}

This is a list of supplementary files associated with this preprint. Click to download.

- supplementarymaterial.pdf 